\title{
Tracing the Region of Interest in Thermal Human Face for Respiration Monitoring
}

\author{
Farah AL-Khalidi \\ College of Science / Computer Science \\ Department \\ Al-mustansiriya University \\ Baghdad, Iraq. \\ Heather Elphick \\ Consultant in Pediatric Respiratory \\ Medicine, Sheffield Children's NHS \\ Foundation Trust, \\ Sheffield, U.K.
}

\author{
Reza Saatchi \\ Reader in Computer Engineering \& DSP, \\ Faculty of ACES, Sheffield Hallam University \\ Sheffield, U.K.
}

\author{
Derek Burke \\ Consultant in Pediatric Accident and \\ Emergency Medicine, Sheffield Children's \\ NHS Foundation Trust \\ Sheffield, U.K.
}

\begin{abstract}
Monitoring respiration rate, i.e. the rate air is inhaled and exhaled is an important indicator of an individual's health. Respiration rate is generally measured using sensors attached to the patient's body.

These contact based methods have a number of limitations, for example the attached sensor can cause discomfort to the patients.

A novel, automated, non-contact based method of respiration monitoring, based on thermal imaging of the skin surface centered on the tip of the nose for the nose breathing as well as the mouth region for the mouth breathing. These methods are developed as well as the Image processing techniques were used to enhance the thermal images, remove unwanted noise and segmented the ROI.

In this study the shape and size of the region of interest (ROI) are investigated. The ROI represents the facial affected area most affected by exhaled air temperature changes. This area is the tip of the nose and the upper lip for the nose breathing and the mouth area for the mouth breathing. Segmenting the ROI was considered an important task in monitoring respiration by thermal imaging.
\end{abstract}

Further work is in progress to enhance the algorithm so that it can cope with very large head movements.

Keywords: Non-contact respiration monitoring, region of interest, thermal imaging processing .

\section{INTRODUCTION}

The respiratory system supplies an adequate amount of oxygen to the body to facilitate energy production and facilitates a suitable acid-base status by removing unwanted carbon dioxide from it. Respiration rate is an important clinical indicator of an individual's health [1]. Mazzanti et al[2] have reported that respiration rate is an important parameter in intensive care monitoring as well as being a parameter influencing the prescribed drugs.

Instruments exist to accurately monitor a variety of other physiological information such as body temperature, heart rate, and blood pressure.
However existing devices for monitoring breathing have been developed but many provide only an estimate of breathing rate due to the complexities associated with measuring this physiological parameter. Conventional methods commonly require physical contact with the patient's body. These include nasal or oronasal thermistor which measures changes in the temperature of exhaled air [3], air pressure transducers [4,5], exhaled $\mathrm{CO} 2$ [6], respiratory sounds analysis $[7,8]$ and impedance plethysmography [5,9]. Abdominal strain gauge transducer [10] . Respiratory rates can also be derived from the ECG [11, 12, and 13]. These methods have limitations including sensor displacement, deadspace caused by requirement for facemasks and alterations in breathing itself caused by the attachment of a sensor to the subject.

In clinical practice, the most commonly used method of respiratory rate monitoring is visually by observing the patient. This is subjective and can thus be inaccurate.

Recently, a new infrared (IR) imaging method has been proposed to monitor human breathing and to compute the respiratory rate by fast Fourier transform and statistical methods [14, 15, and 16]. However the requires of these methods that the subject avoid movement.

\section{METHODOLOGY}

An advanced thermal camera (FLIR A40) that has a thermal sensitivity of $0.08^{\circ}$ Kelvin was used for the study. The camera was fixed on a tripod in front of the patent at a distance of about one meter. The camera settings were: emissivity $0.92^{\circ}$, reflected temperature $15^{\circ} \mathrm{C}$ and relative humidity $50 \%$. Images were recorded at 50 frames per second. This produced 6000 thermal images over the two minute duration (i.e. 120 seconds x 50 images). The recorded images were processed off-line using the Matlab image processing toolbox. The respiration "region of interest" (ROI) tracking method described by Al Khalidi et al. [17] was used and refined to accommodate head movement during recording.

A median lowpass filter of size five was used to reduce unwanted noise A median lowpass filter of size five was used to reduce unwanted noise, The Sobel edge detection scheme [18] was used to identify the boundary of the subjects' heads in the thresholded images. The Sobel masks were: 


$$
\begin{array}{cc}
\text { Row Mask } & \text { Column Mask } \\
G_{x}=\left[\begin{array}{ccc}
-1 & -2 & -1 \\
0 & 0 & 0 \\
1 & 2 & 2
\end{array}\right], \quad G_{y}=\left[\begin{array}{ccc}
-1 & 0 & 1 \\
-2 & 0 & 2 \\
-1 & 0 & 1
\end{array}\right]
\end{array}
$$

The warmest facial region from the part of the image that had been segmented from its background was determined. This area represented one of the two areas between the bridge of nose and the inner of the eyes. This area was marked. The process was repeated to indentify the second warmest facial region from the part of the image that had been segmented from its background. This area also represented the other of the two areas between the bridge of nose and the inner of the eyes. The lowest average facial temperature from the part of the image that had been segmented from its background below the warmest regions was determined. This corresponded to the tip of the nose.

To deal with limitation of this method, the modification has been investigated.

Since the thermal image enhance by median low pass filter of size five, The images were thresholded to separate the human head from the image background. This operation was performed by considering the facial temperature distribution. The temperature of the image background was relatively lower than the temperature of a subject's head. A suitable threshold was $30{ }^{\circ} \mathrm{C}$, and therefore this operation was performed as,

$$
g(x, y)= \begin{cases}0 & \text { if } \quad f(x, y)<30 \\ f(x, y) & \text { if } \quad f(x, y) \geq 30\end{cases}
$$

Where $f(x, y)$ and $g(x, y)$ represent image pixels prior and after the thresholding operation respectively.

The Sobel edge detection scheme was used to identify the boundary of the subjects' heads in the thresholded images.

In order to select the image area covered by the subject's face, an ellipse was automatically superimposed on the filtered images by the developed software. The location and size of the ellipse were determined as follows:

- $\quad$ The highest (xmax) and lowest (xmin) pixels' locations of the head boundary in the vertical direction were identified and the centre between these two locations (x0) was determined.

- Centred at $\mathrm{x} 0$, the head boundary points in the horizontal direction was identified, providing ymin and ymax.. Then, the centre $(\mathrm{y} 0)$ between ymin and ymax was calculated. The diagonals of the ellipse (i.e. $2 \mathrm{a}$ and $2 \mathrm{~b}$ ) were determined, where $\mathrm{a}$ and $\mathrm{b}$ were calculated from $\mathrm{x} 0$, and $\mathrm{y} 0$ to $\mathrm{xmin}$ and ymin respectively.

The position of the ellipse on the filtered images was then determined by the software using the ellipse equation,

$$
\frac{\left(x_{i}-x_{0}\right)^{2}}{a^{2}}+\frac{\left(y_{i}-y_{0}\right)^{2}}{b^{2}}=1
$$

Fig.1. shows the position of the ellipse on an image.

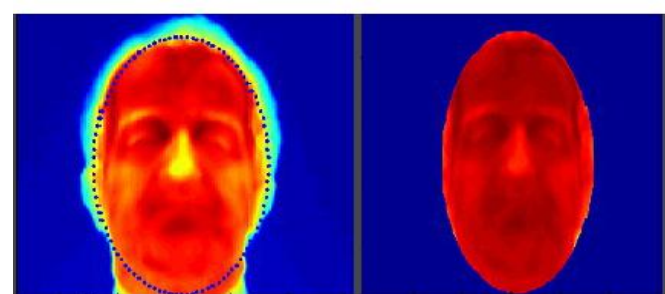

Fig. 1. The position of the ellipse superimposed on the filtered thermal image

Initially the image area enclosed by the ellipse was scanned to identify the warmest and coolest region. This was to aid selection of the area most affected by respiration through the nose especially when there were large head movements. The warmest region corresponded to a small area between the bridge of nose and the inner of an eye. The coolest region corresponded to an area centered on the tip of the nose. A circle was placed on the identified coolest region in such a way that it covered the tip of the nose and the upper lip. The reason for identifying the warmest region first then the coldest region underneath was to reduce the possibility of a wrong region being chosen, especially when there were large head movements. The circled region, the "region of interest" (ROI) was then used to obtain respiratory signal.

This method worked well when the subject breathed through the nose (not mouth) and the mouth remained closed. An open mouth became the warmest facial region, causing the method to fail with some images, because the algorithm works only when the mouth is closed. Therefore, this problem (opened mouth) was dealt by only looking for the warmest region on the upper part of the ellipse thus excluding the mouth region. The scanning was performed beneath the identified warmest area and the coldest area was located. The lowest value within this region was identified. This corresponded to the tip of the nose. The circle was centred on the tip of the nose, as shown in Fig.2.

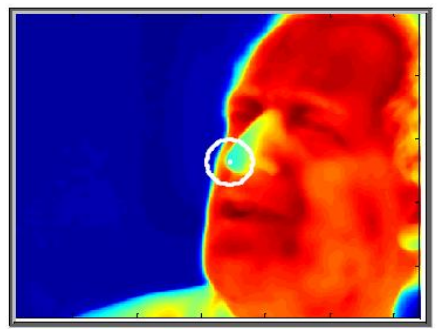

Fig. 2. The ROI : the position of ROI on the mouth.

The circled area represents the ROI for extracting the respiratory signal. This region was first divided into eight equal concentric segments as shown in Fig.3.

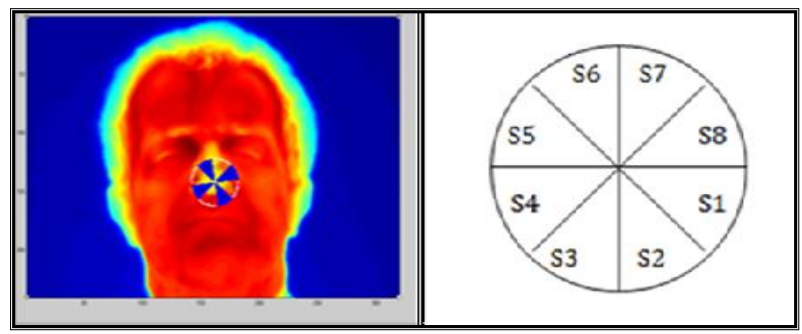

Fig. 3. : The ROI : (a) the position of ROI on the tip of the nose, (b) the eight segments of ROI. 
This allowed for a more detailed temperature analysis. The pixel values within each segment were averaged to obtain a single value representing that segment. The process was repeated for each of the 6000 images recorded from each subject. The averaged pixel values of the eight segments were then separately plotted against time to produce eight respiratory signals. In order to determine the respiration rate automatically, the respiration signals were digitally filtered using a $5^{\text {th }}$-order Butterworth filter with cut-off frequency of 1 Hz. This cut-off frequency was sufficiently low for the respiration signal to be smoothed for further processing. It was sufficiently high to allow 60 cycles per minutes to be detected. The peak-to-peak time interval of each respiration cycle ( $\mathrm{T}$ seconds) was determined by the software. The respiration rate (in cycles per minute) was then determined by first producing an average of respiration cycles. Then, the reciprocal of this value was multiplied by 60 to obtain respiration rate in cycles per minute [19]. This process is illustrated in Fig. 4 below.

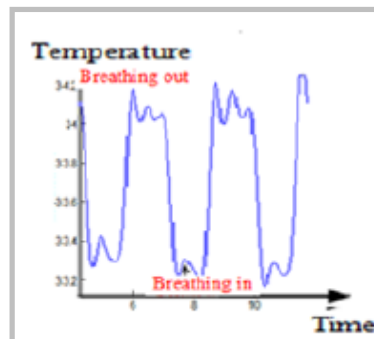

a

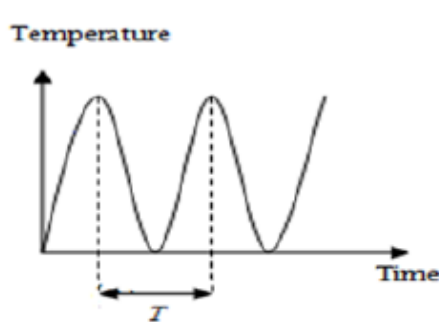

$\mathrm{b}$

Fig. 4. The respiration signal. (a) The fluctuations immediately after the signal level changes are consistent in pattern in different respiration cycles. (b) Its filtered signal indicating the respiration (T).

\section{RESULTS AND DISCUSSION}

One of the main reasons for inaccurate information for tracking images with large diagonal head movements was using a rectangular shape for the ROI as shown in Fig.5
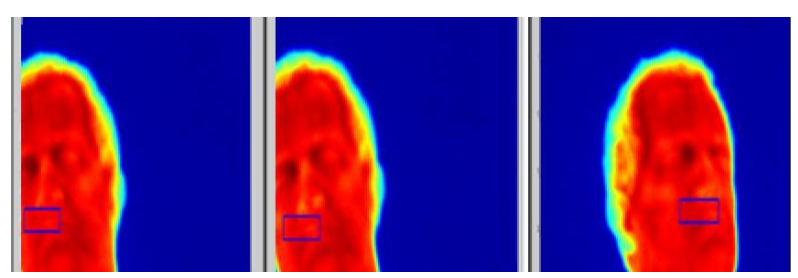

Fig. 5. : The rectangular representation of the ROI with large head movements

Another problem with using a rectangular shape for the ROI is that the rectangular area always fill outside the boundary of the images in which the nose appeared to be too close to the edge as shown in Fig.6
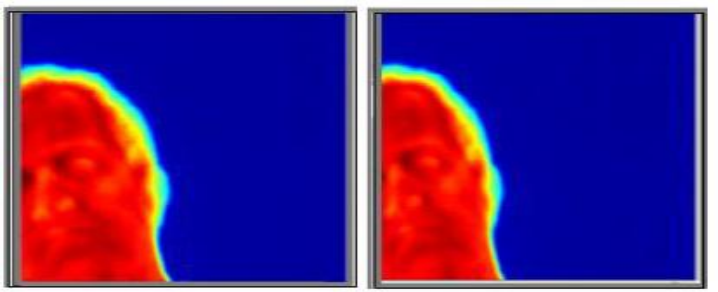

Fig. 6. : A head movement highlighting the nose very close to the edge of the image
As a results, we used a circle as a ROI to fix all the issues that we mentioned before which included the diagonal head movement and the nose appearing too close to the edge of the image. Fig.7 shows the ROI being represented by a circle.
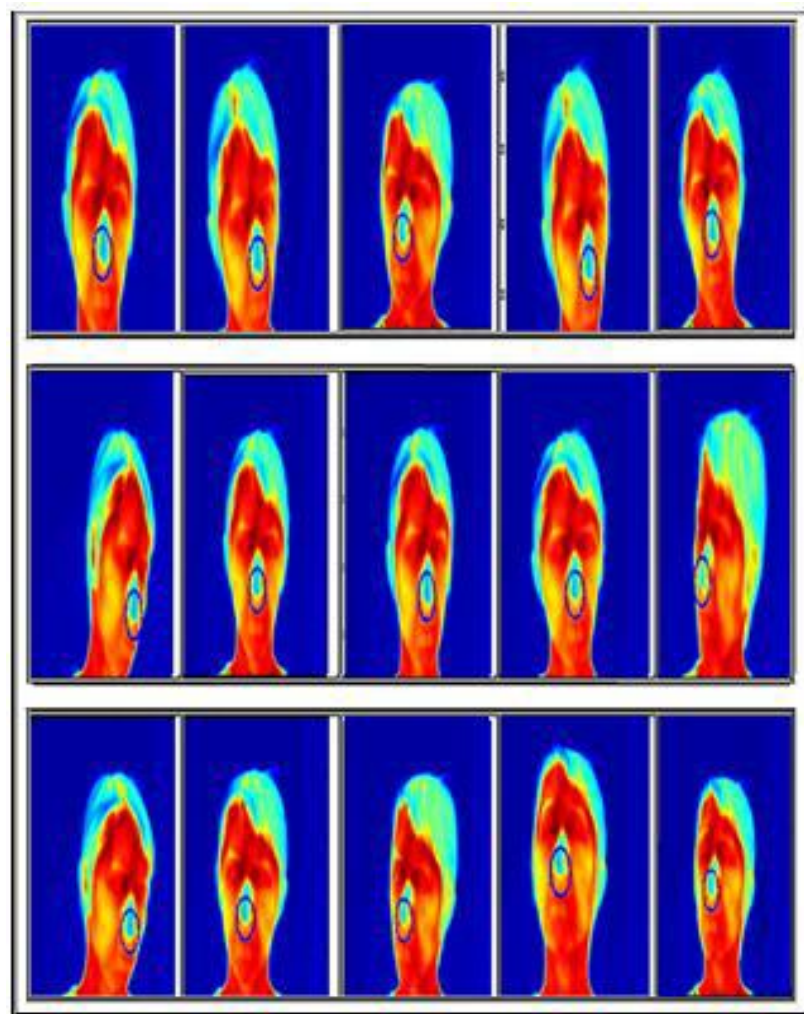

Fig. 7. : The ROI highlighted by a circle.

Table 1 provides an evaluation of the modification of tracking method. The number of images that the ROI had not successfully tracked was visually determined. The accuracy of the tracking methods was calculated by determining the percentages of images that had not successfully tracked the ROI.

TABLE 1. TRACKING ANALYSIS RESULTS FOR DIFFERENT HEAD MOVEMENT TYPES

\begin{tabular}{|c|c|c|c|c|}
\hline Subject & $\begin{array}{c}\text { Number } \\
\text { of } \\
\text { image }\end{array}$ & $\begin{array}{c}\% \text { failure in } \\
\text { the first } \\
\text { tracking } \\
\text { method }\end{array}$ & $\begin{array}{l}\% \text { failure in } \\
\text { the } \\
\text { modification } \\
\text { of tracking } \\
\text { method }\end{array}$ & Video type \\
\hline SO.01 & 6000 & 2.3 & 0 & Random movement \\
\hline SO.02 & 6000 & 0.68 & 0 & Random movement \\
\hline SO.03 & 6005 & 0.2 & 0.02 & Random movement \\
\hline SO.04 & 6009 & 0 & 0 & Regular movement \\
\hline SO.05 & 5991 & 0 & 0 & Regular movement \\
\hline SO.06 & 5991 & 0 & 0 & Random movement \\
\hline SO.07 & 6000 & 0.69 & 0.04 & Random movement \\
\hline SO.08 & 6010 & 0.03 & 0 & Random movement \\
\hline SO.09 & 6000 & 0 & 0 & Random movement \\
\hline SO.10 & 6000 & 0 & 0 & Random movement \\
\hline SO.11 & 6005 & 0.1 & 0.03 & Random movement \\
\hline SO.12 & 5991 & 0 & 0 & Random movement \\
\hline SO.13 & 6010 & 0 & 0 & Regular movement \\
\hline SO.14 & 5991 & 0.21 & 0 & Regular movement \\
\hline SO.15 & 5991 & 0 & 0 & Random movement \\
\hline SO.16 & 1148 & 0 & 0 & Random movement \\
\hline SO.17 & 2550 & 0 & 0 & Random movement \\
\hline SO.18 & 5991 & 0 & 0 & Regular movement \\
\hline SO.19 & 5991 & 0 & 0 & Random movement \\
\hline
\end{tabular}




\begin{tabular}{|l|l|l|l|l|}
\hline SO.20 & 1000 & 0.12 & 0 & Random movement \\
\hline SO.21 & 1000 & 0.17 & 0 & Random movement \\
\hline SO.22 & 550 & 0.13 & 0.02 & Random movement \\
\hline SO.23 & 500 & 0 & 0 & Random movement \\
\hline SO.24 & 50 & 0 & 0 & Random movement \\
\hline SO.25 & 50 & 0 & 0 & Regular movement \\
\hline
\end{tabular}

The tracking was considered as a failure when the ROI had not been located successfully for an image. The proportion of tracking failure was calculated as the total number of failures divided by the total number of images in a recorded video. Failed tracking was caused either by the subjects in the ROI extending too far from the image or by the effect of the environment on body temperature.

Fig. 8 shows the respiration plots obtained by averaging the pixel values of each segment of the ROI highlighted in Fig.3. The clarity of respiration signals obtained from the segments varied, with segments 3 and 7 providing the most clear signal and segment 1 providing the least clear signal. This result indicates that for respiration monitoring it would be advisable to consider all the eight segments and then selecting the segment that provides best respiration signal.

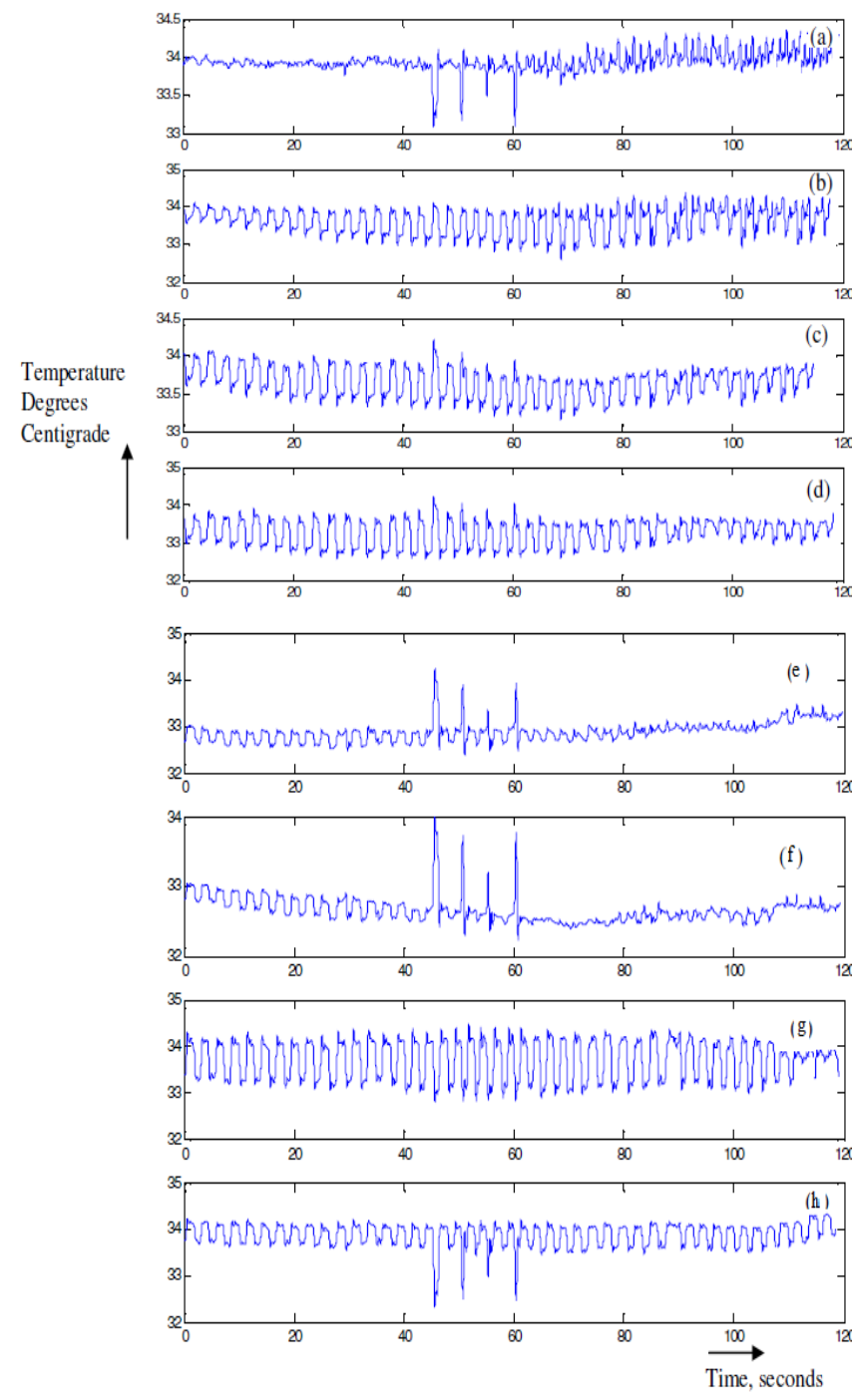

Fig. 8. :Respiration signal from segments 1 to 8. (a) segment 1, (b)segment 2, (c) segment 3 (d) segment 4, (e)segment 5 (f) segment 6, (g)segment 7 (h) segment 8.
Fig.9 shows the signal produced by averaging the pixels from the complete ROI. The respiration cycles appear distorted, confirming the need to partition and process the different parts of the ROI separately

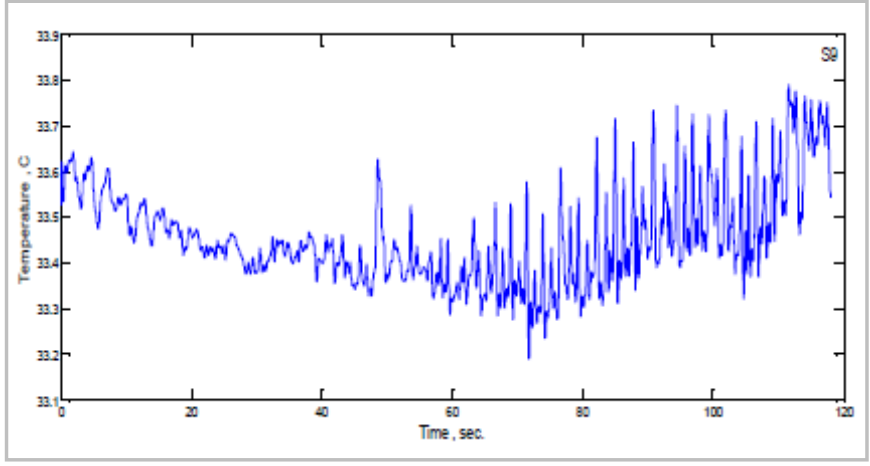

Fig. 9. : Respiration signal obtained by averaging pixel values from the complete ROI

\section{THE SIZE OF THE ROI}

To investigate the accuracy of the respiration rate measurement according to the size of the ROI, the circle radius was initially set to

$$
R_{\text {circle }}=\frac{1}{16} \times N
$$

Where $N$ is the number of image rows. Value was reduced by increasing the value in Equation 1 from 16 to 17 , and increased by decreasing the value in Equation 1, from 16 to 15. Circle R In each case the average pixel value within each circle was plotted against image number (see Fig.10). The results indicated changing the ROI by the specified amounts does not make a significant change to plots produced as they cover the nostrils.

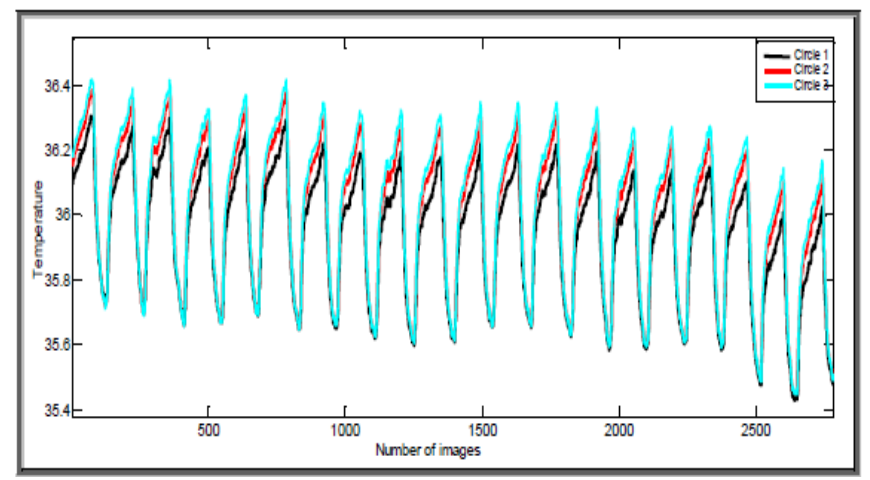

Fig. 10. : Average pixel value within ROI represented by different circle sizes.

\section{CONCLUSIONS}

A method to specify the shape and size of the region of interest (ROI) are investigated. The ROI represents the facial affected area most affected by exhaled air temperature changes. This area is the tip of the nose and the upper lip in thermal images for nose breathing.

The shape of the ROI robustly dealt with static, regular and random head movements. During recordings under different head movement types it was possible to successfully detect the ROI even with the mouth opened as circle associated with the tracking the ROI and respiration process.

The processing was off-line due to the extent of image processing involved. 
Further work on optimizing the image and signal processing will enable measurements to take place in real time. There were further issues with signal detection during large head movements or when subjects wore glasses. Further improvements are currently being sought to deal with these limitations. A customized, portable, inexpensive device for measurement of respiratory rate is under development.

\section{REFERENCES}

[1] J. Fieselmann , S. Hendryx , C. Helms., D. Wakefield, "Respiratory rate predicts cardiopulmonary arrest for internal medicine patients", The Journal of General Internal Medicine, vol.8, pp.354-360. , (1993).

[2] B. Mazzanti, C. Lambert and J. de Bie, "Validation of an ECG-drived respiration monitoring method", Computers in Cardiology, vol.30, pages 613-616. (2003).

[3] K. Storck , M. Karlsson, P. Ask and D. Loyed, "Heat transfer evaluation of the nasal thermistor technique" , IEEE Transactions on Biomedical Engineering, vol. 43, no. 12, pp. 1187-1191, (1996).

[4] M. Shneerson . Sleep Medicine: A Guide to Sleep and its Disorders, Handbook Second edition. Blackwell Publishing Ltd UK, ( 2005).

[5] L. Lee-Chiong, Editor. Sleep: a comprehensive handbook, Wiley (2006).

[6] M. Folke, L. Cernerud , M. Ekstro, B. Hok . Comparative provocation test of respiratory monitoring methods. Journal of Clinical Monitoring and Computing,;17(2):97-103,(2002).

[7] J. Werthammer, J. Krasner, J. DiBenedetto and R. Stark, (1983), "Apnea Monitoring by Acoustic Detection of Airflow", Pediatrics vol.71 pp,53-55.

[8] P. Corbishley, Rodriguez-Villegas. Breathing detection: toward a miniaturised, wearable, battery-operated monitoring system. IEEE Transactions on Biomedical Engineering.55 (1):196:204,( 2008).

[9] A. Blom. Monitoring of respiration and circulation. CRC Press LLC , Boca Raton London New York Washington, D.C(2004).

[10] K. Nepal, E. Biegeleisen and T. Ning, ”Apnea detection and respiration rate estimation through parametric modeling", Proceedings of the $28^{\text {th }}$ IEEE Annual Northeast Bioengineering Conference, Philadelphia, Pennsylvania. pp.277-278, (2002).

[11] S. Ding, X. Zhu, W. Chen, D. Wei , Derivation of respiratory signal from single-channel ECGs based on source Ding statistics, International Journal of Bioelectromagnetism, 6(1):43-49,(2004).

[12] G. Furman , Z. Shinar , A. Baharav , S. Akselrod Electrocardiogram derived respiration during sleep, Computers in Cardiology; 32:351-354,(2005)

[13] B. Moody , G. Mark , A. Bump ,S. Weinstein , D Berman ,E. Mietus and L. Goldberger , "Clinical validation of ECG-derived respiration (EDR) technique" IEEE Computers in Cardiology, vol.13, pp.507-510., (1986).

[14] C. Eveland , D. Socolinsky and L. Wolff ,"Tracking human faces in infrared video", Image and Vision Computing, vol.21 pp.579-590, July (2003).

[15] R. Murthy and I. Pavlidis , "Non-contact monitoring of respiratory function using infrared imaging," in IEEE Engineering in Medicine and Biology Magazine.vol.25,pp.57-57., (2006).

[16] J. Fei , Z. Zhu and I. Pavlidis , "Imaging respiratory rate in the $\mathrm{CO} 2$ absorption band," in Proceedings of the 27th Annual International Conference of the IEEE Engineering in Medicine and Biology Society, (Shanghai, China), pp.700-590, September 1-4. (2005)

[17] F. AL-Khalidi , R. Saatchi , B. Burke and H. Elphick "Tracking Human Face Features in Thermal Images for Respiration Monitoring " ACS/IEEE International Conference on Computer Systems and Applications in Tunisia , May 16-19th .( 2010)

[18] R.Gonzales , R. Woods and L. Eddins, ."Digital image processing using MATLAB", Pearson Education, the United States of America. (2004).

[19] R.Saatchi , F. AL-Khalidi , B. Burke and H. Elphick "Thermal Image Analysis of the Skin Surface Centred on the Tip of the Nose for Respiration Monitoring" The ICEDSP International Conference on Computer Systems and Applications in India. December $10-11^{\text {th }} 2009$. (2009). 